\title{
Effects of Geometric Parameters on Dynamic Stability of the Annular Flow-shell System
}

\author{
Wen-Bo NINGa, Yun XU, Ying-Hua LIAO, Zhi-Rong LI \\ a School of Mechanical Engineering, Sichuan University of Science and Engineering, Zigong \\ 643000, People's Republic of China
}

Keywords: Cylindrical shell, Stability, Annular flow, Gap ratio.

\begin{abstract}
Considering their particular geometric structures, the stator and rotor cans in canned motor reactor coolant pump are assumed to be elastic coaxial cylindrical shells in this paper. An ideal, incompressible, swirling fluid flows the annulus between the inner shell (can) and the outer shell (can). Thin shell structures such as cans are prone to buckling instabilities under fluid forces. Furthermore, a lot of accidents were caused by losing stability. Dynamic stability analysis of the stator can subjected to helical annular flow is presented by means of Donnell-type shell equations and the potential flow theory. Specially, the effects of the geometrical parameters of the annular flow-shell system are studied. The results show that the critical velocities decrease with the crease of the values of the length-to-radius and radius-to-thickness. Moreover, the effects of annular gaps and boundary conditions on the stability of annular flow-shell system are given.
\end{abstract}

\section{Introduction}

Studies on dynamic behaviors of coaxial cylindrical shells subjected to annular flow are extensive as result of such structural elements application in many engineering systems. Tijsseling [1] presented reviews related to the development of fluid-structure interaction in liquid-filled pipe systems. A more detailed review on the stability and dynamics of pipes and shells conveying fluid was presented by Païdoussis [2, 3] in the form of a two-volume book. Dynamics and stability of coaxial cylindrical shells conveying inviscid or viscous fluid were studied with the aid of Fourier-transform techniques [4-6]. It was found that the annular flow velocity of losing stability is lower than the value of internal flow velocity and viscous forces destabilize the system in the annular space between the two shells. The effects of system parameters on stability were also discussed. Kzragiozis et al. [7] studied the effect of geometry on stability of clamped cylindrical shell subjected to internal flow. Considering unsteady viscous forces, Chebair et al. [8,9] preformed further studies on the same problem theoretically and experimentally. Based on the semi-membrane theory, an approximate theory of annular-flow-induced instability of coaxial cylindrical shells was presented [10, 11]. Chen and Bert [12] considered dynamic stability of isotropic or composite-material cylindrical shell containing a swirling fluid flow by using travelling-wave method. The obtained results showed that the fluid rotation severely decreases the stability of the shell-fluid system. The dynamic behaviors of coaxial cylindrical shells subjected to annular fluid flow with having both the axial and tangential velocity components were given by Bochkarev and Matveenko [13, 14]. They found that the combined action of both velocity components affects the stability of the system essentially.

In this paper, the stator and rotor cans in reactor coolant pump are treated as thin-walled shells due to their particular structures. The stator can subjected to annular flow is studied only due to lacking for supporters resulting from instability easier. The present study is a continuation of the previous theoretical work [15], which aims to investigate the influence of geometric parameters on the dynamic stability of shells conveying fluid. The effects of different boundaries on the stability of shells are compared. 


\section{Problem Formulation}

The system under consideration consists of two coaxial cylinders of length $L$ as shown in Figure. 1. The inner shell with the radius of $r_{i}$ is considered to be perfectly rigid. The outer circular cylindrical shell with the radius of the middle surface ${ }^{r}$, the wall thickness $h_{o}$ is located concentrically. The coaxial cylinders are considered in an orthogonal coordinate system $(O ; r, \theta, x)$, in which the origin $\mathrm{O}$ is located on the left end at the middle point of the centre-body. $\mathrm{x}, \theta$, and $\mathrm{r}$ are in the axial, circumferential, and radial direction, respectively. The same material properties of the two cylinders are characterized by elastic modulus $E$, Poission' ratio $v$, and density $\rho_{s}$. The fluid in the annular space between the two shells is considered to be incompressible with the density $\rho_{f}$, flowing in X-, $\theta$ - directions with velocity components $U_{o}, \Omega_{o}$.

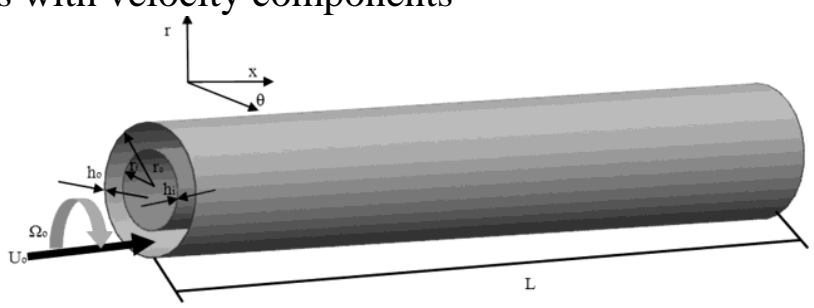

Figure 1. Schematic diagram of the coaxial cylindrical shells under consideration.

\section{The Equations of Motion}

Based on the classic Donnell shell motions [15], the motion equations of an inner or outer presressed shell incorporating axial loads can be written in one linear matrix operator:

$$
\zeta\left[\begin{array}{l}
u_{i / o} \\
v_{i / o} \\
w_{i / o}
\end{array}\right]=\left[\begin{array}{l}
0 \\
0 \\
p
\end{array}\right]
$$

where the subscripts $\mathrm{i}$ indicates the inner shell and o pertains to the outer shell. $\mathrm{u}, \mathrm{v}$ and $\mathrm{w}$ are the displacements of the middle surface point of a shell along $\mathrm{x}, \theta$ and $\mathrm{r}$ directions respectively. $p$ represents the unsteady inviscid force acting on the shells per unit area.

$$
\zeta=\frac{E h}{1-v^{2}}\left[\begin{array}{lll}
\zeta_{11} & \zeta_{12} & \zeta_{13} \\
\zeta_{21} & \zeta_{22} & \zeta_{23} \\
\zeta_{31} & \zeta_{32} & \zeta_{33}
\end{array}\right]
$$

where

$$
\begin{aligned}
& \zeta_{11}=\frac{\partial^{2}}{\partial x^{2}}+\frac{1-v}{2 r^{2}} \frac{\partial^{2}}{\partial \theta^{2}}-\rho_{s} \frac{1-v^{2}}{E} \frac{\partial^{2}}{\partial t^{2}}, \zeta_{12}=\zeta_{21}=\frac{1+v}{2 r} \frac{\partial^{2}}{\partial x \partial \theta}, \zeta_{13}=\zeta_{31} \frac{v}{r} \frac{\partial}{\partial x}, \zeta_{22}=\frac{1-v}{2} \frac{\partial^{2}}{\partial x^{2}}+\frac{1}{r} \frac{\partial^{2}}{\partial \theta^{2}}-\rho_{s} \frac{1-v^{2}}{E} \frac{\partial^{2}}{\partial t^{2}}, \\
& \zeta_{33}=\frac{1}{r^{2}}+\frac{h^{2}}{12} \Delta \Delta+\rho_{s} \frac{1-v^{2}}{E} \frac{\partial^{2}}{\partial t^{2}}, \Delta=\frac{\partial^{2}}{\partial x^{2}}+\frac{1}{r^{2}} \frac{\partial^{2}}{\partial \theta^{2}}
\end{aligned}
$$

The unsteady inviscid force $\mathrm{p}$ which is associated with shell motions is determined by the potential flow theory. Using the impermeable boundary conditions, the unsteady inviscid fluid force on the inner shell or outer shell is given by 


$$
\begin{aligned}
& p_{i}=-\frac{\rho_{f}}{\Gamma}\left(\begin{array}{l}
\frac{\partial}{\partial t}+ \\
\frac{V_{o}}{R} \frac{\partial}{\partial \theta}+U_{o} \frac{\partial}{\partial x}
\end{array}\right)^{2} \\
& \left\{\begin{array}{l}
{\left[I_{n}\left(\lambda r_{i}\right) K_{n}^{\prime}\left(\lambda r_{o}\right)-I_{n}^{\prime}\left(\lambda r_{o}\right) K_{n}\left(\lambda r_{i}\right)\right] w_{i}} \\
+\left[I_{n}^{\prime}\left(\lambda r_{i}\right) K_{n}\left(\lambda r_{i}\right)-I_{n}\left(\lambda r_{i}\right) K_{n}^{\prime}\left(\lambda r_{i}\right)\right] w_{o}
\end{array}\right\}
\end{aligned}
$$

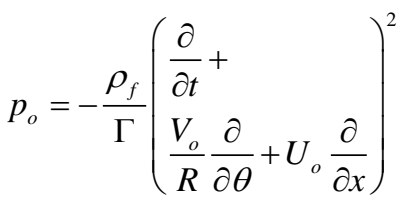

$$
\begin{aligned}
& \left\{\begin{array}{l}
{\left[I_{n}\left(\lambda r_{o}\right) K_{n}^{\prime}\left(\lambda r_{o}\right)-I_{n}^{\prime}\left(\lambda r_{o}\right) K_{n}\left(\lambda r_{o}\right)\right] w_{i}} \\
+\left[I_{n}^{\prime}\left(\lambda r_{i}\right) K_{n}\left(\lambda r_{o}\right)-I_{n}\left(\lambda r_{o}\right) K_{n}^{\prime}\left(\lambda r_{i}\right)\right] w_{o}
\end{array}\right\}
\end{aligned}
$$

where ${ }^{I_{n}}$ and $K_{n}$ are the modified Bessel functions of the first and second kind and order $\mathrm{n}$. The prime denotes the differentiation and $\lambda$ indicates the axial wavenumber. Also, $\mathrm{t}$ stands for time.

in which $\Gamma=I_{n}^{\prime}\left(\lambda r_{i}\right) K_{n}^{\prime}\left(\lambda r_{o}\right)-I_{n}^{\prime}\left(\lambda r_{o}\right) K_{n}^{\prime}\left(\lambda r_{i}\right)$.

The present paper is concerned with dynamics and stability of a single cylindrical shell, the expressions $3 \mathrm{a}$ and $3 \mathrm{~b}$ can be simplified.

$$
\begin{aligned}
p_{i}= & -\frac{\rho_{f}}{\Gamma}\left(\frac{\partial}{\partial t}+\frac{V_{o}}{R} \frac{\partial}{\partial \theta}+U_{o} \frac{\partial}{\partial x}\right)^{2} \\
& {\left[I_{n}\left(\lambda r_{i}\right) K_{n}^{\prime}\left(\lambda r_{o}\right)-I_{n}^{\prime}\left(\lambda r_{o}\right) K_{n}\left(\lambda r_{i}\right)\right] w_{i} } \\
p_{i}= & -\frac{\rho_{f}}{\Gamma}\left(\frac{\partial}{\partial t}+\frac{V_{o}}{R} \frac{\partial}{\partial \theta}+U_{o} \frac{\partial}{\partial x}\right)^{2} \\
& {\left[I_{n}\left(\lambda r_{i}\right) K_{n}^{\prime}\left(\lambda r_{o}\right)-I_{n}^{\prime}\left(\lambda r_{o}\right) K_{n}\left(\lambda r_{i}\right)\right] w_{i} }
\end{aligned}
$$

\section{Method of Solution}

Consider an inner or outer shell with simply supported(SS) or clamped supported (CC) boundary conditions. The boundary conditions at $\mathrm{x}=0, \mathrm{~L}$ are given

$$
\begin{aligned}
& \text { SS: } v_{i / \mathrm{o}}=w_{i / \mathrm{o}}=N_{x}=M_{x}=0 \\
& \mathrm{CC}: u_{i / \mathrm{o}}=v_{i / \mathrm{o}}=w_{i / \mathrm{o}}=\frac{\partial w_{i / \mathrm{o}}}{\partial x}=0
\end{aligned}
$$

Displacement components $\mathrm{u}, \mathrm{v}$ and $\mathrm{w}$ of an inner or outer shell with different boundary conditions can be expanded in the form of travelling wave.

$$
\begin{aligned}
& \left.\left[\begin{array}{l}
u_{i / o} \\
v_{i / o} \\
w_{i / o}
\end{array}\right]=\left[\begin{array}{l}
u_{m n} \exp [-i(\lambda x+n \theta-\omega t)] \\
v_{m n} \exp [-i(\lambda x+n \theta-\omega t)] \\
w_{m n} \exp [-i(\lambda x+n \theta-\omega t+\pi / 2)]
\end{array}\right]_{(\mathrm{SS},} \lambda=\frac{m \pi}{L}\right) \\
& {\left[\begin{array}{l}
u_{i / o} \\
v_{i / o} \\
w_{i / o}
\end{array}\right]=\left[\begin{array}{l}
u_{m n} \exp [-i(\lambda x+n \theta-\omega t)] \\
v_{m n} \exp [-i(\lambda x+n \theta-\omega t)] \\
w_{m n} \exp [-i(\lambda x+n \theta-\omega t+\pi / 2)]
\end{array}\right]_{(\mathrm{CC},}^{\left.\lambda=\frac{(2 m+1) \pi}{2 L}\right)}}
\end{aligned}
$$

where $\omega$ is frequency.

The flowing quantities are introduced to make the dimensionless analysis. 


$$
\begin{gathered}
\tau=r_{i} \sqrt{\frac{\rho_{s}\left(1-v^{2}\right)}{E}, \bar{t}=\frac{t}{\tau}, \bar{h}=\frac{h}{r_{i}}, \bar{x}=\frac{x}{r_{i}}, \bar{L}=\frac{L}{r_{i}}, \bar{r}=\frac{r}{r_{i}}, \quad \overline{F_{r}}=\frac{\tau^{2}}{\rho_{s} r_{i}^{2}} F_{r}, \overline{U_{o}}=\frac{U_{o} \tau}{r_{i}}, \lambda_{c}=\frac{r_{o}}{r_{i}}, k=\frac{r_{o}-r_{i}}{r_{i}}, \bar{\omega}=\omega \tau,} \\
{\left[\begin{array}{l}
\bar{u} \\
\bar{v} \\
\bar{w}
\end{array}\right]=\frac{1}{r_{i}}\left[\begin{array}{l}
u_{i / o} \\
v_{i / o} \\
w_{i / o}
\end{array}\right]}
\end{gathered}
$$

Substituting eqs. (3), (6) into eq. (1), considering the inner cylindrical body is perfectly rigid, eq. (1) is reformulated as in the dimensionless form.

$$
\begin{gathered}
\zeta\left[\begin{array}{c}
\overline{u_{o}} \\
\overline{v_{o}} \\
-\overline{i w_{o}}
\end{array}\right]=\left[\begin{array}{l}
0 \\
0 \\
0
\end{array}\right] \\
\zeta=\left[\begin{array}{lll}
\zeta_{11} & \zeta_{12} & \zeta_{13} \\
\zeta_{21} & \zeta_{22} & \zeta_{23} \\
\zeta_{31} & \zeta_{32} & \zeta_{33}
\end{array}\right] \\
\zeta_{11}=-\lambda^{2}-\frac{1-v}{2 \lambda_{c}^{2}} n^{2}+\bar{\omega}^{2}, \zeta_{12}=\zeta_{21}=\frac{1+v}{2 \lambda_{c}} \lambda n, \zeta_{13}=\zeta_{31}=-\frac{i v \lambda}{\lambda_{c}}, \zeta_{22}=-\frac{1-v}{2} \lambda^{2}-\frac{n^{2}}{\lambda_{c}^{2}}+\bar{\omega}^{2}, \\
\zeta_{33}=\frac{1}{\lambda_{c}^{2}}+\frac{\bar{h}^{2}}{12}\left(\lambda^{2}+\frac{n^{2}}{\lambda_{c}^{2}}\right)^{2}-\bar{\omega}^{2}-\frac{\bar{\Gamma}_{2}}{\bar{\Gamma}} \frac{1}{\bar{h}} \frac{\rho_{f}}{\rho_{s}}\left(\bar{\omega}-\lambda \overline{U_{o}}-n \frac{V_{o}}{\lambda_{c}}\right)^{2}
\end{gathered}
$$

The determinant of the operator matrix must equal to zero because of the existence of nontrivial solutions of eq. (7).

$$
|\zeta|=0
$$

The left hand of eq. (9) is a polynomial of variable $\bar{\omega}$. The dispersion relation is evaluated by zero-level contour method. For a given value of $\bar{V}_{o}\left(\right.$ or $\left.\bar{U}_{0}\right)$, the contour plot of $\bar{\omega}_{\text {versus }} \overline{U_{0}}\left(\right.$ or $\left.\bar{V}_{0}\right)$ is realized by the method. Avoiding cumbersome Fourier transforms, the critical points of losing stability are obtained readily.

\section{Results and Discussion}

In this case the inner shell is considered perfectly rigid, dynamics and stability of the outer shell conveying fluid are analyzed, having the axial and/or circumferential velocity components. The results obtained by using zero-level contour method are validated [15].

In each subsection below, materials properties adopted are:

$$
E_{o}=2.0 \times 10^{11} \mathrm{P}_{\mathrm{a}}, v=0.3, \rho_{s}=8870 \mathrm{Kgm}^{-3}, \rho_{f}=1000 \mathrm{Kgm}^{-3} .
$$

Geometric parameters are given by

$$
L=1.5 m, r_{o}=0.2825 m, h_{o}=3.81 \times 10^{-4} m, k=\frac{r_{o}-r_{i}}{r_{i}}=\frac{1}{10} .
$$

\section{The Effect of Geometric Parameters}

In order to investigate effects of different length-radius -ratios ${ }^{L / r_{o}}$ on the critical flutter velocity $U_{o}$ with different boundary conditions, the relation curves of $U_{o}-L / \mathrm{r}_{o}$ are plotted in figure 2 . It is 
clear that increasing ${ }^{L / r_{o}}$, the critical velocity lowers rapidly. In other words, the increase of the shell length degrades the stability of the annular flow-shell system.

Figure 3 shows the effect of the ratios ${ }^{r_{o}} / h_{o}$ on the stability of the system. It is found that the critical velocity lowers rapidly decreases remarkably with the increase of ratios ${ }_{o} / h_{o}$. So, the more the shell is thick, the more the shell is stable.

From the figures 3, 4, the critical axial velocities of clamped shell are much more the corresponding values of simply supported shell.

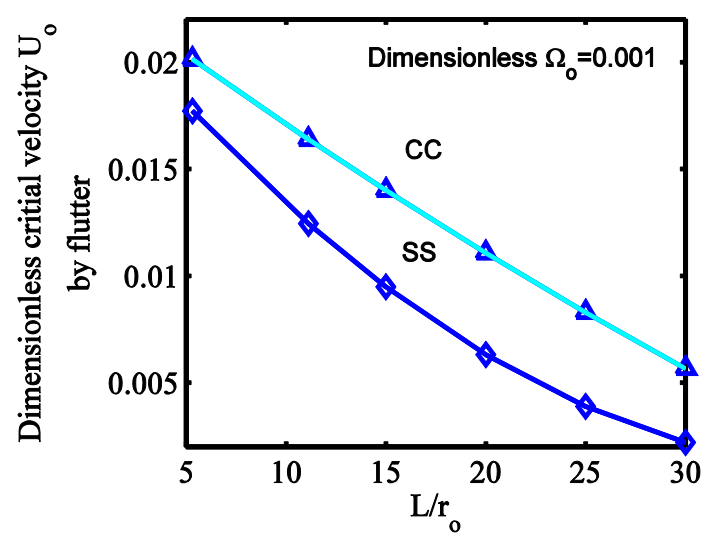

Figure 2. Effects of different $L / \mathrm{r}_{o}$ ratios on critical axial velocities.

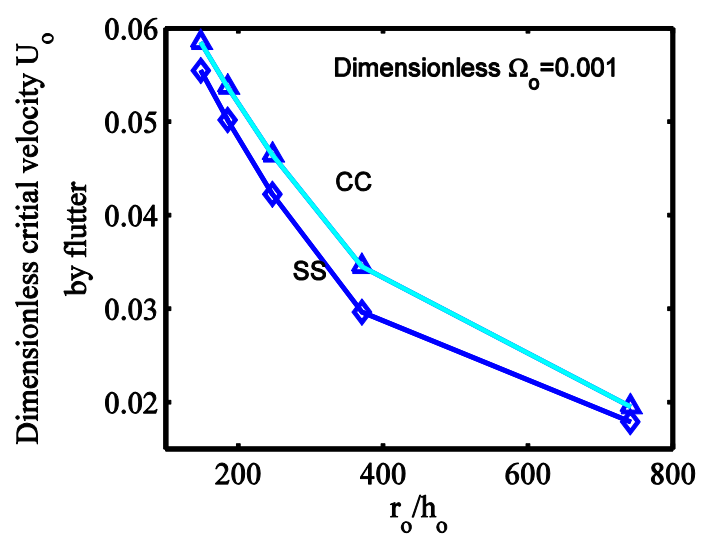

Figure 3. Effects of different ${ }^{\mathrm{r}_{o}} / h_{o}$ ratios on critical axial velocities.

\section{The Effect of Annular Gap}

Figure 4 shows the effects of different annular gap ratios on the critical axial velocity. Without considering the axial velocity, the effects of different annular gap ratios on the critical angular velocity are shown in figure 5. Note that the annular gap ratio $\mathrm{k}$ is a function of the variable ${ }^{r_{i}}$ here, whereas the outer radius ${ }^{r_{o}}$ keeps constant. From the two figures, it is seen that the critical velocities increase with increasing the gap ratios. 


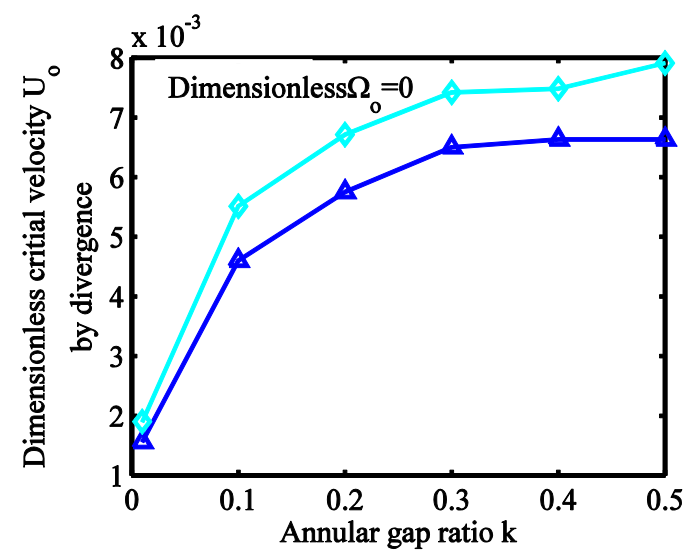

Figure 4. Effects of different gap ratios $k$ on critical axial velocities.

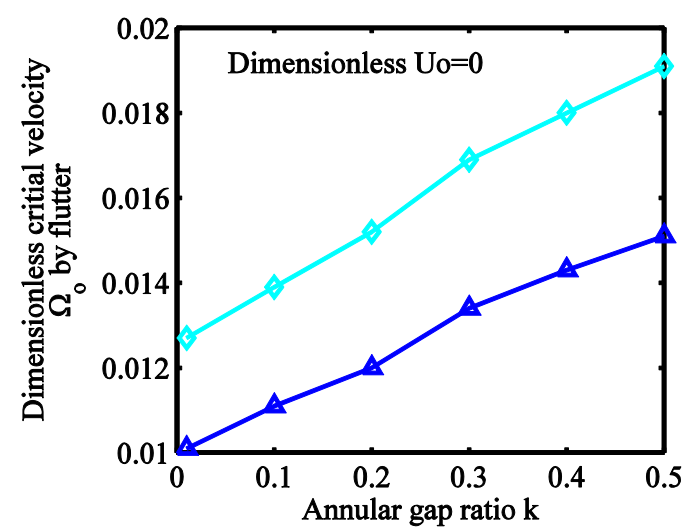

Figure 5. Effects of different gap ratios ${ }^{k}$ on critical angular velocities.

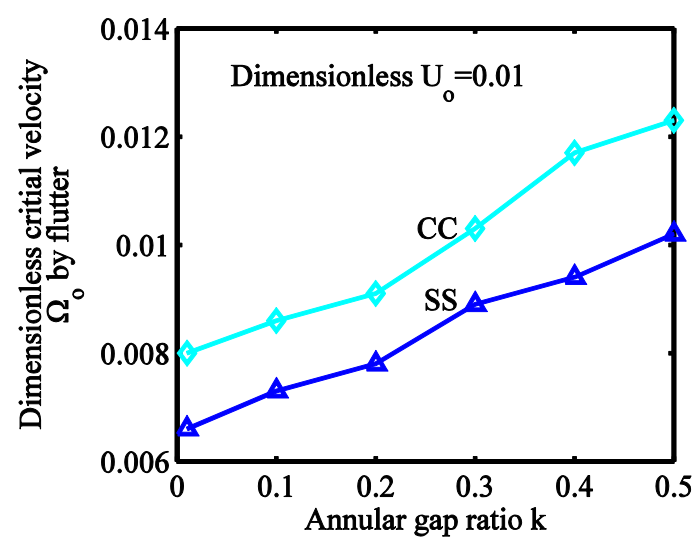

Figure 6. Effects of different gap ratios ${ }^{k}$ on critical angular velocities.

Taking account into the combined action of the two velocity components, figure 6 shows the similar results, i.e. the critical angular velocity decreases rapidly with the increase of the gap ratio.

\section{Conclusions}

Dynamic stability of the stator can (shell) in canned motor reactor coolant pump which is subjected to annular flow has been investigated based on the Donnell shallow theory and linear potential flow in this paper. The effects of important parameters such as shell thickness, shell length, annular gap on the stability of the annular flow-shell system are evaluated. 
The results obtained show that with with the increase of ratios $\mathrm{r}_{o} / h_{o}, L / \mathrm{r}_{o}$, the critical velocity decreases. The results also show that the effects of variants of annular gap ratio on the critical axial velocity are more obvious than the angular velocity.

It has been found that clamped shells are more stable than the simply supported shells.

\section{Acknowledge}

The work is supported by grants from the Training Program of Sichuan University of Science and Engineering (Grant No. 2014PY04) and the Talents Introductions Project of Sichuan University of Science and Engineering (Grant No.2016RCL01). The authors are grateful for the financial support.

\section{References}

[1] A. S. Tijsseling, Fluid-structure interaction in liquid-filled pipe systems, J. Fluids Struct. 10, 2 (1996)

[2] M. P. Païdoussis, Fluid Structure Interaction Vol. 1, (Academic Press, London, 1998)

[3] M. P. Païdoussis, Fluid Structure Interaction Vol. 2, (Elsevier Academic Press, London, 2003)

[4] M. P. Païdoussis, S. P. Chen and A. K. Misra, Dynamics and stability of coaxial cylindrical shells containing flowing fluid, J. Sound Vib. 97, 2 (1984)

[5] M. P. Païdoussis, A. K. Misra and S. P. Chen, Dynamics and stability of coaxial cylindrical shells conveying viscous fluid. J. Appl. Mech. 52, 2 (1985)

[6] M. P. Païdoussis, A. K. Misra and V. B. Nguyen, Internal- and annular-flow-induced instabilities of a clamped-clamped or cantilevered cylindrical shell in a coaxial conduit: The effects of system parameters, J. Sound Vib. 159, 2 (1992)

[7] K. Z. Karagiozis, M. P. Paidoussis, M. Amabili, Effect of geometry on the stability of cylindrical clamped shells subjected to internal fluid flow, Comput Struct 85, 11-14 (2007)

[8] E. I. Chebair, A. K. Misra and M. P. Païdoussis, Theoretical study of the the effect of unsteady viscous forces on inner- and annular-flow-inducted instability of cylindrical shells, J. Sound Vib. 138, 3 (1990)

[9] E. I. Chebair, M. P. Païdoussis and A. K. Misra, Experimental study of annular-inducted-instabilities of cylindrical shell, J. Fluids Struct. 3, 4 (1989)

[10]J. Horáĉek, Approximate theory of annular flow-induced instability of cylindrical shells, J. Fluids Struct, 7, 2 (1993)

[11]J. Horáceek and I. Zolotarev, Influence of fixing the edges of a cylindrical shell with conveying fluid on its dynamic characteristics, Int. Appl. Mech. 20, 8 (1984)

[12]T. L. C. Chen, C. W. Bert, Dynamic stability of isotropic or composite-material cylindrical shell carrying a rotation flow by using travelling wave forms, J. Appl Mech 44, 1 (1977)

[13] S. A. Bochkarev and V. P. Matveenko, Stability of a cylindrical shell subjected to an annular flow of rotating fluid, J. Sound Vib. 332, 18 (2013)

[14] S. A. Bochkarev and V. P. Matveenko, Stability analysis of cylindrical shells containing a fluid with axial and circumferential components, J. Appl. Mech. Tech. Phys. 53, 5 (2012)

[15] W. B. Ning, D. Z. Wang, J. G. Zhang, Dynamics and stability of a cylindrical shell subjected to annular flow including temperature effects, Arch. Apll. Mech. 86, 4 (2016) 